\title{
Moraxella canis
}

National Cancer Institute

\section{Source}

National Cancer Institute. Moraxella canis. NCI Thesaurus. Code C86525.

A species of aerobic, Gram negative, cocci shaped, bacteria assigned to the phylum

Proteobacteria. This species is positive for oxidase, catalase, butyrate esterase, and y-

glutamyl-aminopeptidase activities, negative for phenylalanine deaminase, urease, lipase

and lecithinase activity and relatively halointolerant. M. canis is an upper-airway

commensal of dogs and cats and is considered nonpathogenic in humans. 